\title{
Association of Atlanto-Occipital Dislocation, Retroclival Hematoma, and Hydrocephalus: Management and Survival in a Pediatric Patient
}

\author{
Isaac L. Lee ${ }^{1} \quad$ Luis F. Vasquez $^{2} \quad$ Alan H. Tyroch ${ }^{1}$ \\ ${ }^{1}$ Department of Surgery, Texas Tech University Health Sciences \\ Center El Paso, El Paso, Texas, United States \\ 2 Department of Neurosurgery, University Medical Center El Paso, \\ El Paso, Texas, United States
}

\author{
Todd T. Trier ${ }^{2}$
}

J Neurol Surg Rep 2017;78:e46-e51.

\begin{abstract}
Keywords

- atlanto-occipital dislocation

- retroclival hematoma

- hydrocephalus

- pediatric trauma
\end{abstract}

\author{
Address for correspondence Isaac Lee, MD, MPH, Department of \\ Surgery, Texas Tech University Health Sciences Center El Paso, \\ 4800 Alberta Avenue, El Paso, TX 79905, United States \\ (e-mail: isaac.l.lee@ttuhsc.edu).
}

\section{Introduction}

Diagnosed atlanto-occipital dislocations (AOD) are rare, survival of such an injury even more so. However, AOD is becoming more of a survivable injury due to timely diagnosis and improved management of such injuries in the prehospital and hospital setting. ${ }^{1}$ Despite such improvements, AOD remains an injury with high morbidity and mortality, often due to early complications. ${ }^{2}$ Survival of patients presenting with a triad of AOD, retroclival hematoma, and hydrocephalus have been described in a case series by Klimo et al. ${ }^{3}$ In this case report, we describe and discuss a patient who survived this triad through early recognition and treatment, combining clinical suspicion with utilization of current recommendations.

\section{Case Report}

A 4-year-old male patient presented to the trauma center after a motor vehicle crash 1 hour before arrival. The driver of the car lost control after hitting an embankment. Subse-

quently, there was a head-on collision with another vehicle. The patient was an unrestrained passenger in the back seat sitting on another passenger's lap. He was not ejected from the vehicle. According to his parents, he lost consciousness with no seizure activity. He was confused en route to the trauma center. No nausea or vomiting was noted. The patient reported hitting his head and was complaining of neck pain. He had no significant medical history.

Vital signs upon presentation to our trauma center were: blood pressure, 78/40 mm Hg; heart rate, 141 beats/min; respiratory rate, 18 breaths/min; and the temperature, $37.6^{\circ}$ C. Glasgow Coma Score was 14 upon presentation (E4-V4-M6). Physical examination revealed equal and reactive pupils, no initial tenderness to palpation over the cervical spine, contusions over the submandibular area and the anterior neck, and an abrasion over the manubrium. He had no visible trauma on the head. No bony step-offs were palpated over the entire spine. No neurological deficits were elicited. No other signs of injury were found aside from a contusion on the right arm.

Initial imaging included radiographs of the chest and pelvis, along with computed tomography (CT) imaging of his head,

(c) 2017 Georg Thieme Verlag KG Stuttgart · New York
License terms

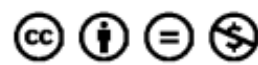

October 21, 2016 accepted after revision

February 10, 2017
DOI http://dx.doi.org/ 10.1055/s-0037-1600914 ISSN 2193-6358. 


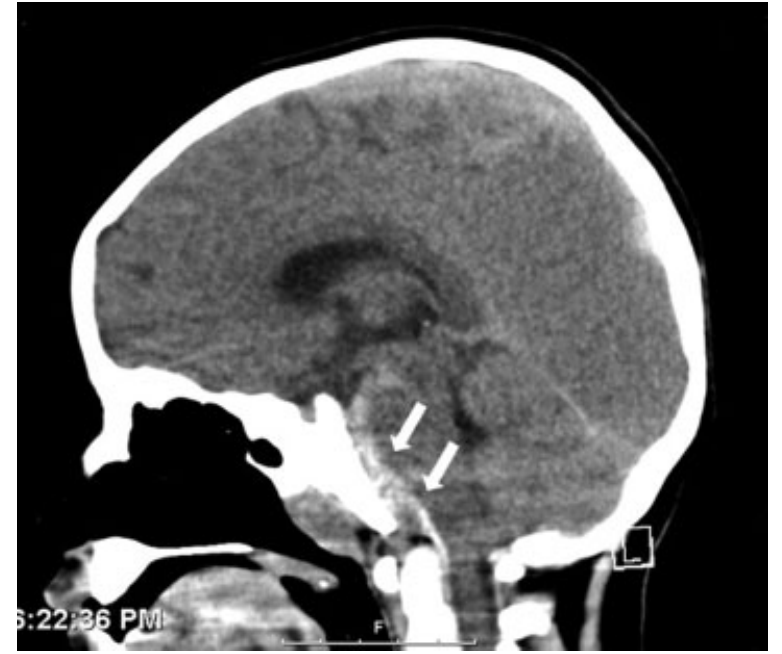

Fig. 1 Initial head CT demonstrating the presence of the retroclival hematoma. CT, computed tomography.

thorax, abdomen, cervical/thoracic/lumbar spine, and a CT angiogram of the neck. Subarachnoid hemorrhage in the prepontine cistern was found along with bleeding around the foramen magnum (-Fig. 1), with no cervical bony or ligamentous injury. He also had a minimally displaced avulsion fracture of the lateral epicondyle of the right humerus.

Neurosurgery was consulted for management of the subarachnoid hemorrhage. The main initial concern was that the patient had a borderline distraction of the dens and concern for atlantoaxial ligamentous injury. The patient's ventricles were dilated as well. He was admitted to the pediatric intensive care unit (PICU), and the patient was kept in spine precautions with an Aspen collar. Magnetic resonance imaging (MRI) of the brain and cervical spine was obtained on the second hospital day. The MRI showed craniocervical distraction with retroclival hemorrhage (-Fig. 2). There was stripping of the poster-

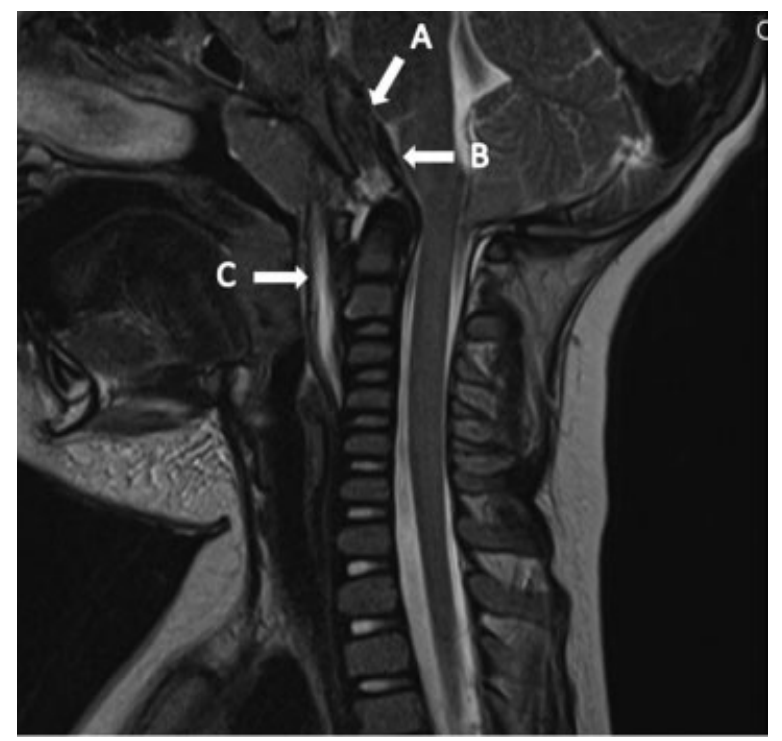

Fig. 2 T2-weighted MRI showing a retroclival hematoma (A), stripping of the tectorial membrane (B), and prevertebral hemorrhage $(C)$. MRI, magnetic resonance imaging. ior longitudinal ligamentous complex with subligamentous hemorrhage along the base of the clivus. There was also separation of the anterior longitudinal ligament from the ventral margin of the clivus with prevertebral hemorrhage. The tip of the dens was displaced posteriorly and longitudinally in relation to the basion, causing marginal compression upon the cervicomedullary junction. The predental space was normal $(2 \mathrm{~mm})$, indicating intact cruciate ligaments. There was no evidence of disc protrusion or cervical cord injury. Bilateral intraventricular hemorrhage was also present with mild lateral ventricular dilatation suggesting communicating hydrocephalus.

The patient was kept in spine precautions and remained in the PICU. On the fourth hospital day, he experienced worsening headaches. No neurological deficits were noted at this time. Repeat CT of the head showed persistent hydrocephalus, a stable third ventricle but increased dilatation of the lateral ventricles and lateral horns (-Fig. 3). A ventriculostomy was placed in the right lateral ventricle. A repeat $\mathrm{CT}$ of the head on the subsequent day demonstrated good interval decompression of the ventricles after ventriculostomy placement.

On the seventh hospital day, he underwent an occipitalC1-C2 fusion with right and left transarticular C1-C2 screws along with allograft and arthrodesis of the occiput and $\mathrm{C} 1-\mathrm{C} 2$. Intraoperative fluoroscopy confirmed the neutral position of the neck. Decortication of the occiput, $\mathrm{C} 1$, and C2 was performed and an allograft was placed for firm fixation for the hardware. An occipital plate was placed, as well as two screws. Each screw crossed the lateral masses of $\mathrm{C} 1$ and the medial edges of the $\mathrm{C} 2$ pedicle. Two $25 \mathrm{~mm}$ rods with a slight bend secured the plate to the screws. No operative complications were encountered. Cervical spine CT confirmed achievement of gross anatomic alignment.

The ventriculostomy remained in place until the tenth hospital day. After ventriculostomy removal, no evidence of herniation, midline shift, or hydrocephalus was found on CT imaging. The prepontine subarachnoid hemorrhage was nearly completely resolved. The child was discharged on

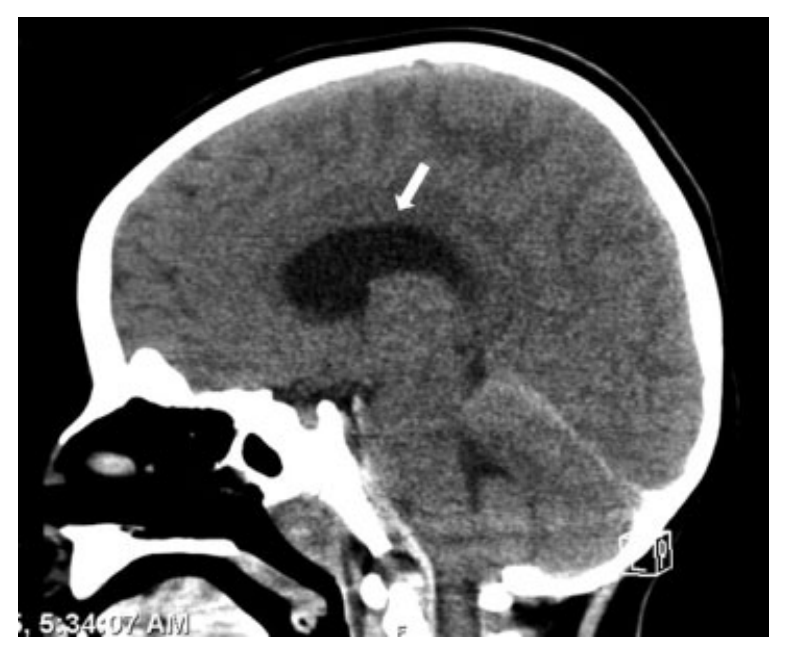

Fig. 3 Head CT on hospital day 4 showing the presence of hydrocephalus. CT, computed tomography. 


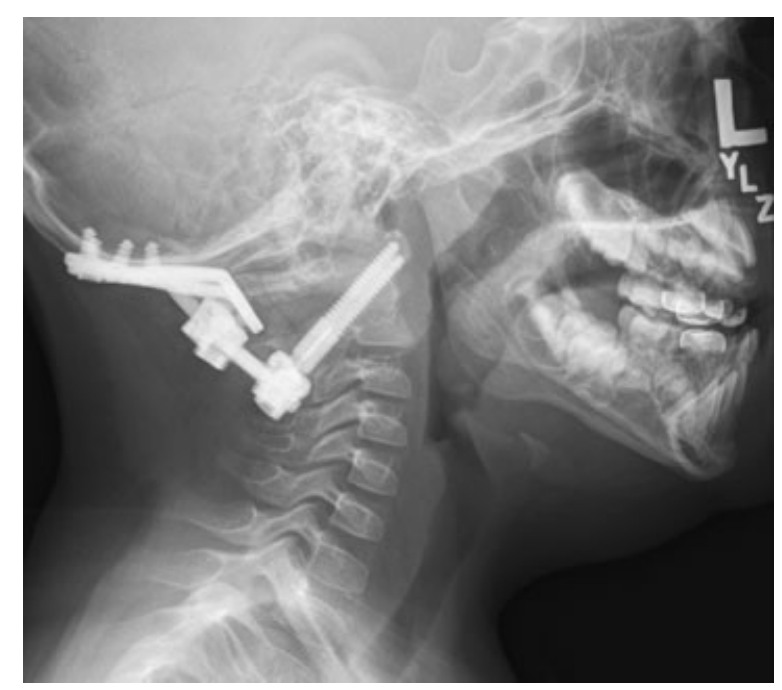

Fig. 4 Three month poststabilization cervical spine radiograph.

the $11^{\text {th }}$ hospital day, 4 days after his cervical fusion. He was instructed to wear the Aspen collar until his neurosurgery clinical appointment a month after discharge. He had no deficits in activity or limitations in the diet at the time of discharge. Subsequent visits at 3 and 6 months were unremarkable ( - Fig. 4), and he made a full recovery from his atlanto-occipital injuries.

\section{Discussion}

\section{Presentation}

The triad of atlanto-occipital dislocation, retroclival hematoma, and secondary hydrocephalus was first described by Vera et al. ${ }^{4}$ The mechanism of injury of AOD is a sudden deceleration which causes the head to be thrown forward. ${ }^{5}$ The neurological injury may be a result of direct traction or compression of the spinal cord. The injury may also arise indirectly from vascular injury causing ischemia. ${ }^{1}$ Signs of AOD include headaches, ataxia, other motor or sensory neurological deficits, and respiratory distress. ${ }^{5}$ The cranial nerves, as well as any part of the spinal cord at the craniocervical junction (CCJ), may be affected. ${ }^{1}$ Symptoms range from unilateral weakness to quadriplegia. AOD is classified into four categories according to Traynelis et al:

- Type 1: Anterior dislocation of the head relative to the spine

- Type 2: Longitudinal dislocation

- Type 3: Posterior dislocation

- Other type: Other types of dislocation such as rotational, lateral, and multidirectional ${ }^{6}$

Retroclival hematomas are rare, occurring mostly as a result of high energy trauma involving the head or neck. ${ }^{7}$ Posttraumatic retroclival hematomas have been reported primarily in the pediatric population. ${ }^{8}$ Symptoms mirror those of AOD, caused by compression of the spinal cord and brainstem from the hematoma. Secondary to the retroclival hematoma in our patient was the communicating hydrocephalus. Presentation of hydrocephalus includes symptoms such as nausea, vomiting, deficits in coordination, impaired gait, and urinary incontinence.

\section{Anatomy}

AOD is more common in pediatric trauma compared with adult trauma due to biomechanical and anatomic factors. ${ }^{9}$ These factors include a high head-to-body weight ratio, incomplete development of neck musculature, increased ligamentous laxity, and horizontal facet joints. ${ }^{5}$ The atlanto-occipital joint consists of the superior articular facets of the atlas, the occipital condyles, the articular capsule, and the ligamentous/membranous tissues in between them. ${ }^{1}$ The $\mathrm{CCJ}$ is stabilized mainly through several ligaments attaching the cranium to the cervical spine. Likewise, the dens is kept in place in relation to the atlas through ligaments. The anterior atlanto-occipital membrane is a continuation of the anterior longitudinal membrane; it connects the anterior arch of the atlas to the anterior portion of the clivus. The tectorial membrane is a continuation of the posterior longitudinal ligament which continues superiorly. ${ }^{7}$ It passes posterior to the odontoid process and attaches to the inferior portion of the clivus. ${ }^{1}$ The alar ligaments connect the lateral aspects of the odontoid process to the medial occipital condyles. The apical ligament attaches the tip of the dens to the basion. The cruciate ligament has a superior, transverse, and inferior band located posterior to the dens. The superior and inferior bands connect the dens to the basion, and the transverse band stabilizes the dens to the lateral masses of the atlas.

Retroclival hematomas may occur secondary to the stripping of the tectorial membrane. ${ }^{7}$ Injury to the anterior longitudinal ligament may also result in a hematoma in the retropharyngeal space. Disruption of the anterior longitudinal ligament can result in prevertebral hemorrhage, as was seen in our case. Compression of the spinal cord from the hematoma and the AOD led to a communicating hydrocephalus.

\section{Diagnosis}

It has been challenging to develop a measurement standard for diagnosis of AOD using current standards because these standards rely on distances and relationships of occipital structures to the cervical spine. ${ }^{10}$ As the skull can be highly mobile in AOD, the true mobility and relationship between the cranium and the cervical spine may not be captured during the moment of imaging. Thus, diagnosis can be easily overlooked due to the confounding factor of patient positioning. ${ }^{11}$ This can alter landmarks and possible hide joint dislocation. Stabilization of the spine may hide AOD as well. ${ }^{2}$ Anomalies of pediatric cervical spine anatomy that may be misinterpreted as an injury include an absence of the cervical lordosis, a large atlantodens interval, pseudosubluxation of C2 and C3, a wide retropharyngeal space, anterior wedging of the cervical vertebral bodies, incomplete ossification of the odontoid process, and open synchondroses. ${ }^{5}$

The atlanto-occipital interface is not easily appreciated using cervical spine plain film, making this modality a poor choice in diagnosing AOD. Delays in diagnosis may lead to serious complications of AOD and avoidable morbidity and 
mortality. The use of computed tomography affords a clearer view of the atlanto-occipital joint as compared with X-ray. The standard for evaluation of the atlanto-occipital joint is MRI, with its capability to measure soft tissue anomalies. However, CT is often used initially in the setting of trauma, and MRI is often unavailable or not feasible; thus the diagnosis of AOD is often made by CT or lateral cervical spine radiograph. Bony displacements of spinal and cranial landmarks have historically been measured using different methods. These diagnostic criteria include the Powers ratio, the basion-dental interval, the basion-axis interval (BAI), the $\mathrm{C} 1-\mathrm{C} 2 / \mathrm{C} 2-\mathrm{C} 3$ ratio, the occiput- $\mathrm{C} 1$ distance, and the Wackenheim clivus line. ${ }^{4}$ In 2013, Pang et al devised the condyle-C1 interval (CCI), which is currently accepted as the diagnostic criteria of choice by the American Association of Neurological Surgeons and the Congress of Neurological Surgeons. ${ }^{12}$

The Powers ratio is a ratio calculated by measuring the distance between the basion and the posterior arch of the atlas and dividing that measurement by the distance between the opisthion and the anterior arch of the atlas. This ratio is less than 1.0 in healthy individuals, a ratio of greater than 1.0 is considered to be diagnostic for AOD. ${ }^{2}$

The basion-dental interval is the distance between the tip of the basion and the tip of the dens. Because children often do not have complete ossification of the dens, this confounding factor affects the interval. As ossification progresses the basion-dental interval decreases; in adults, the normal interval is $5 \mathrm{~mm}$ or less, in children $10 \mathrm{~mm}$ is considered a normal interval. $^{13}$

The BAI requires a line that is extended cephalad from the posterior longitudinal ligament at the dens. The distance from the tip of the basion to this line is the BAI. A normal BAI is less than $12 \mathrm{~mm}$. A BAI greater than $12 \mathrm{~mm}$ suggests anterior dislocation of the cranium. ${ }^{2}$ Unlike the basiondental interval, the BAI is not affected by the state of odontoid ossification.

The $\mathrm{C} 1-\mathrm{C} 2 / \mathrm{C} 2-\mathrm{C} 3$ ratio is based on the interlaminar distances between $\mathrm{C} 1, \mathrm{C} 2$, and $\mathrm{C} 3$. The ratio of the distance between $\mathrm{C} 1$ and $\mathrm{C} 2$ compared with the distance between $\mathrm{C} 2$ and C3 should be less than 2.5. A ratio greater than 2.5 suggests injury to the ligamentum flavum, supporting a diagnosis of $\mathrm{AOD} .^{2}$ The occiput-C1 distance is the distance between the occipital condyles to the $\mathrm{C} 1$ superior facets. ${ }^{2} \mathrm{~A}$ distance greater than $5 \mathrm{~mm}$ is abnormal. ${ }^{2}$ The interval has shown to be the most sensitive for AOD. ${ }^{6}$

The Wackenheim clivus line is extended inferiorly from the angle of the clivus. This line should cross the dens near the tip. ${ }^{2}$ AOD can cause a change in the angle of this line, which is evident on CT, cross-table lateral c-spine X-ray, or MRI. Other radiographic signs of AOD include occipital condyle fracture, hemorrhage, and swelling of the retroclival area. ${ }^{4}$

Retroclival hematomas may be diagnosed with CT imaging, but may be difficult due to beam attenuation caused by the skull base. ${ }^{8}$ MRI is superior to CT in diagnosing retroclival hematomas due to better delineation of soft tissues. In our case (-Fig. 5), the Powers ratio was less than 1.0, the basion-dental interval was 10.3 , which was a borderline normal value. The BAI was 6.4 , which was normal. The

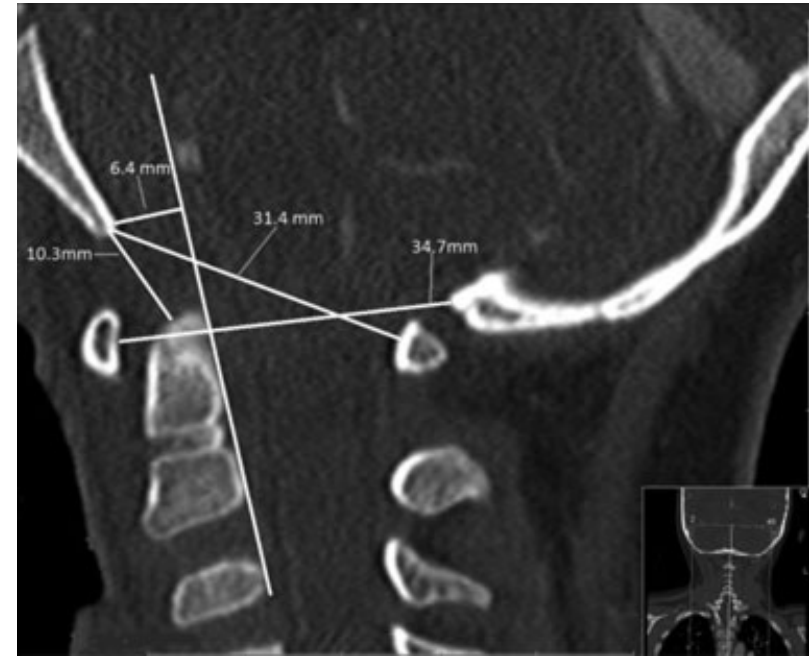

Fig. 5 CT of the cervical spine showing different measurements used to diagnose AOD. AOD, atlanto-occipital dislocation; CT, computed tomography.

C1-C2/C2-C3 ratio was 9.1/5.7, or 1.6. The occiput-C1 distance was $5.7 \mathrm{~mm}$ at the widest points, and the Wackenheim clivus line crossed the dens appropriately. Thus, the only abnormal value was the occiput-C1 distance, which was not taken into account at the time of initial review of the CT images.

The condyle-C1 interval is the mean of measurements taken of the space between the occipital condyles and the C1 superior facets. ${ }^{12}$ Four measurements are taken on the right and left joint spaces using both coronal and sagittal views. The 16 measurements are averaged to produce the combined CCI. Pang et al calculated the mean normal CCI to be $1.28 \mathrm{~mm}$ with a standard deviation of $0.26 \mathrm{~mm}$. The $\mathrm{CCI}$ was also found to be more sensitive and more specific compared with all other radiodiagnostic criteria. ${ }^{14}$ Thus, the American Association of Neurological Surgeons (AANS) and the Congress of Neurological Surgeons (CNS) have adopted this criterion as the current gold standard for diagnosing AOD. ${ }^{15}$ In our patient, the mean CCI was $2.6 \mathrm{~mm}$, meeting the criteria for AOD.(-Fig. 6)

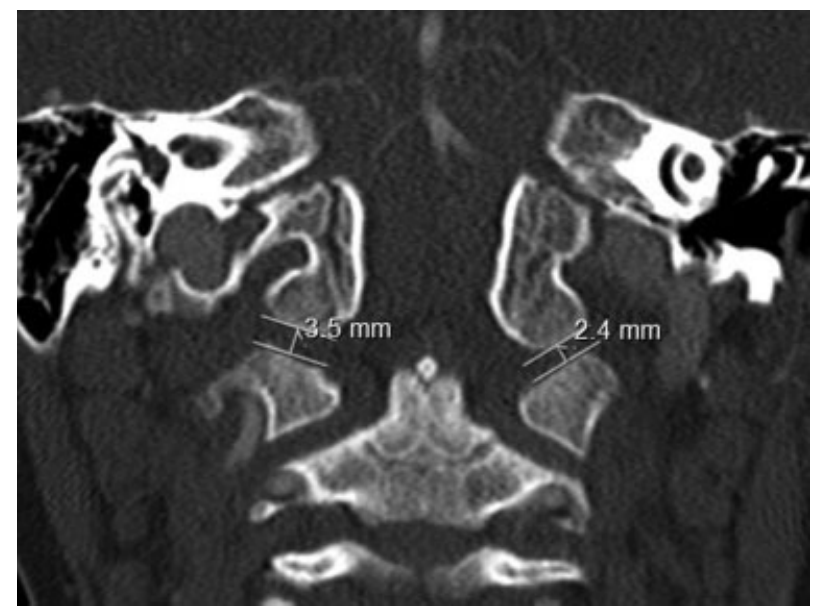

Fig. $6 \mathrm{CT}$ of the c-spine, coronal view, with two of the measurements used for the $\mathrm{CCI}$. CCI, condyle- $\mathrm{C} 1$ interval; $\mathrm{CT}$, computed tomography 


\section{Management}

Treatment of AOD begins with cervical immobilization in patients with suspected craniocervical injury. Surgical fixation and fusion have been described in treating AOD. ${ }^{6}$ Use of traction is not recommended, as the frequency of neurological deterioration is approximately 10\%, 10 times higher than the rate for subaxial injuries. ${ }^{6}$ Treatment with external immobilization alone is not advised, as only a few cases in the literature describe successful outcomes with this treatment alone. Successful management has been reported in children younger than 3 years with halo vest transitioning to Minerva jacket. External immobilization may transition to internal fixation and fusion as the definitive treatment. Posttraumatic retroclival hematomas in the absence of AOD, however, have been successfully treated with cervical immobilization alone. $^{8}$

Management of hydrocephalus primarily consists of diversion of cerebrospinal fluid (CSF) from the brain to other outlets. ${ }^{16}$ A shunt system diverts the flow of CSF to an external collection system or to other parts of the body for absorption. For our case, a ventriculostomy shunted CSF to an external drain. The ventriculostomy was a temporizing measure, and the hydrocephalus resolved after the AOD was treated definitively.

\section{Outcomes}

Atlanto-occipital dislocation has historically been a rare diagnosis made primarily during autopsies, although it is becoming an increasingly survivable injury. ${ }^{1}$ Improvements in the hospital and prehospital care of such injuries play a significant role in increasingly successful outcomes. ${ }^{2}$ Such improvements include areas such as imaging, care on scene, transport services, time to cervical immobilization, and trauma care. ${ }^{2,4}$ Delay of a diagnosis of AOD leads to a higher likelihood of neurological deterioration according to Bellabarba et al. ${ }^{6}$ In their study, a diagnosis of AOD was delayed by a mean of 2 days in trauma patients, and 5 of 13 patients with a delayed diagnosis suffered neurological worsening before AOD was recognized.

In this case, prompt recognition of AOD by MRI led to proper management and survival without significant morbidity. Initial cervical spine CT was unable to detect AOD without CCI criteria, however, borderline distraction of the dens found in the CT image necessitated a timely cervical spine MRI to confirm the diagnosis. The ventriculostomy was adequate for management of the worsening hydrocephalus. The AOD was able to be addressed later in the hospital course, and it was demonstrated that maintaining cervical stabilization with a hard collar is sufficient until definitive treatment is implemented.

\section{Conclusion}

Once a universally fatal diagnosis, AOD is becoming an entity that is survivable without significant morbidity due to advances in radiographic detection and surgical management. We affirm that a favorable outcome can be achieved even in a patient with the sequelae of retroclival hematoma and hydrocephalus. This case also highlights the association of traumatic epidural hematomas with young age. Use of the CCI confirms the diagnosis of AOD, and a high degree of suspicion for a distraction injury in the face of a normal neurological examination also aided in diagnosing and treating the patient adequately. Likewise in the future, a high index of suspicion of a retroclival hematoma is needed in pediatric patients with hyperextension and flexion injuries, especially in those with AOD. We recommend surgical fixation as the definitive treatment of AOD, in line with current AANS and CNS recommendations.

\section{Financial Support}

None.

\section{Note}

The content from this article has not been presented in any form previously.

\section{Conflict of Interest}

The authors report no conflict of interest concerning the materials or methods used in this study or the findings specified in this article.

\section{References}

1 Hall GC, Kinsman MJ, Nazar RG, et al. Atlanto-occipital dislocation. World J Orthop 2015;6(02):236-243

2 Astur N, Sawyer JR, Klimo P Jr, Kelly DM, Muhlbauer M, Warner WC Jr. Traumatic atlanto-occipital dislocation in children. J Am Acad Orthop Surg 2014;22(05):274-282

3 Klimo P Jr, Astur N, Gabrick K, Warner WC Jr, Muhlbauer MS. Occipitocervical fusion using a contoured rod and wire construct in children: a reappraisal of a vintage technique. J Neurosurg Pediatr 2013;11(02):160-169

4 Vera M, Navarro R, Esteban E, Costa JM. Association of atlantooccipital dislocation and retroclival haematoma in a child. Childs Nerv Syst 2007;23(08):913-916

5 Raj D, Rao RD, Smuck M. Orthopaedic Knowledge Update: Spine 4. 4th ed. Rosemont, IL: American Academy of Orthopaedic Surgeons; 2012

6 Theodore N, Aarabi B, Dhall SS, et al. The diagnosis and management of traumatic atlanto-occipital dislocation injuries. Neurosurgery 2013;72(Suppl 2):114-126

7 Agrawal D, Cochrane DD. Traumatic retroclival epidural hematoma - a pediatric entity? Childs Nerv Syst 2006;22(07):670-673

8 Guillaume D, Menezes AH. Retroclival hematoma in the pediatric population. Report of two cases and review of the literature. J Neurosurg 2006;105(4, Suppl):321-325

9 Diaz R, Zouros A, Stewart GM. Atlanto-occipital dislocation with retroclival hematoma in a pediatric patient presenting to the emergency department. Pediatr Emerg Care 2010;26(11): 843-847

10 Horn EM, Feiz-Erfan I, Lekovic GP, Dickman CA, Sonntag VKH Theodore N. Survivors of occipitoatlantal dislocation injuries: imaging and clinical correlates. J Neurosurg Spine 2007;6(02): 113-120

11 Kalani MA, Ratliff JK. Considering the diagnosis of occipitocervical dissociation. Spine J 2013;13(05):520-522

12 Pang D, Nemzek WR, Zovickian J. Atlanto-occipital dislocation: part 1-normal occipital condyle-C1 interval in 89 children. Neurosurgery 2007;61(03):514-521, discussion 521 
13 Wholey MH, Bruwer AJ, Baker HL Jr. The lateral roentgenogram of the neck; with comments on the atlanto-odontoid-basion relationship. Radiology 1958;71(03):350-356

14 Pang D, Nemzek WR, Zovickian J. Atlanto-occipital dislocationpart 2: The clinical use of (occipital) condyle-C1 interval, comparison with other diagnostic methods, and the manifestation, management, and outcome of atlanto-occipital dislocation in children. Neurosurgery 2007;61(05):995-1015, discussion 1015

15 National Guideline Clearinghouse (NGC). Guideline summary: The diagnosis and management of traumatic atlanto-occipital dislocation injuries. In: Guidelines for the Management of Acute Cervical Spine and Spinal Cord Injuries. . Available at: https:// www.guideline.gov/summaries/summary/44301/the-diagnosisand-management-of-traumatic-atlantooccipital-dislocation-injuries-in-guidelines-for-the-management-of-acute-cervicalspine-and-spinal-cord-injuries. Accessed December 16, 2016

16 National Institute of Neurological Disorders and Stroke. Hydrocephalus fact sheet. Available at: https://www.ninds.nih.gov/ Disorders/Patient-Caregiver-Education/Fact-Sheets/Hydrocephalus-Fact-Sheet\#3125_6. Accessed March 25, 2016 